\title{
BRAFV600E Immunohistochemistry in Papillary Thyroid Carcinomas: Relationship Between Clinical and Morphological Parameters
}

\author{
Faruk Erdem KOMBAK ${ }^{1}$ (D), Naziye ÖZKAN ${ }^{2}$ iD, Mustafa Ümit UĞURLU³ ${ }^{\mathbb{D}}$, Handan KAYA \\ 'Department of Pathology, Marmara University, School of Medicine, ISTANBUL, TURKEY, \\ 2Pathology Laboratory Techniques, Marmara University, Vocational School of Health, ISTANBUL, TURKEY \\ ${ }^{3}$ Department of General Surgery, Marmara University, School of Medicine, ISTANBUL, TURKEY
}

The study was presented as a poster at the $29^{\text {th }}$ Congress of the European Society of Pathology, Cologne, Germany.

\begin{abstract}
Objective: To investigate the association of the BRAFV600E mutation with papillary thyroid carcinoma using clinical, morphological and prognostic parameters. We also intend to assess the utility of the BRAFV600E immunohistochemistry and compare it with $B R A F$ polymerase chain reaction (RT-PCR).

Material and Method: We applied BRAFV600E immunohistochemistry in a cohort of 107 papillary carcinomas, 19 adenomas and 13 normal thyroid tissues that was chosen retrospectively between 2011 and 2015. Statistical analysis was based on semiquantitative immunohistochemistry findings. We also applied BRAF RT-PCR in a subgroup of 14 papillary carcinomas, 13 metastatic lymph nodes and 4 adenomas that was chosen randomly.

Results: In regard to the comparison of $B R A F V 600 \mathrm{E}$ immunohistochemistry and $B R A F \mathrm{RT}-\mathrm{PCR}$, a $3+$ nuclear and cytoplasmic immunoexpression was considered 'positive'. The BRAFV600E mutation was most frequently observed in classic variant cases. No mutation was detected in follicular variant cases. The mutational status of the primary tumour and the lymph node metastasis was consistent. A significant relationship of the $B R A F V 600 \mathrm{E}$ mutation was found with prognostic factors such as higher $\mathrm{pT}$ stage, classic variant, lymphatic invasion, perineural invasion, lower mitotic index, lack of tumour capsule, intrathyroidal spread and extrathyroidal extension.

Conclusion: Immunohistochemistry, using the VE1 clone, is a reliable technique for detection of the BRAFV600E mutation. Our results with immunohistochemistry are consistent with a previous effort. In our study, despite the correlation between some pathological prognostic parameters and the $B R A F V 600 \mathrm{E}$ mutation; poor prognosis was found to be irrelevant overall. Morphological parameters seem to be keener than the BRAFV600E mutation. Nevertheless, different series display different results, possibly due to environmental factors. Considering this and the proven success of targeted therapies against the BRAFV600E mutation a thorough assessment would be important.
\end{abstract}

Key Words: Thyroid, Papillary carcinoma, BRAFV600E, Immunohistochemistry, Reverse Transcriptase PCR, Prognosis

\section{INTRODUCTION}

Papillary carcinoma of the thyroid (PTC) is one of the most common cancers with an incidence rate of 14.42 per 100000 person-years in 2010-2013 (1). PTCs have been known to have a better prognosis than other malignant tumours of the human body, although around $10 \%$ exhibit a worse clinical course than expected in PTCs. Several immunohistochemical stains have been used, such as cytokeratin 19 (CK19), Hector Battifora mesothelial cell-1 (HBME-1) and Galectin-3, to diagnose and detect this small group. The rapid development in molecular pathology has led to deeper efforts in coming up with the privileges of targeted therapy options.

(Turk Patoloji Derg 2019, 35:83-91)

Received : 18.07.2018 Accepted : 14.11.2018
The most relevant genetic alterations in PTC are generally mutually exclusive and in the vast majority of cases cause activation of the MAPK pathway; such as BRAF, RET and RAS mutations with $B R A F$ mutations in the centre of attention (2). Mutations affecting the BRAF protooncogene are point mutations, small in-frame deletions, insertions and chromosomal rearrangements; the most frequent of which is the BRAFV600E point mutation. As a group, $B R A F$ mutations activate $B R A F$ kinase and lead to chronic stimulation of the mitogen-activated protein kinase pathway. In PTCs, BRAF mutations have been postulated as a cause of tumour recurrence (3) and worse prognosis $(4,5)$, along with initial tumour pathogenesis. Polymerase

Correspondence: Faruk Erdem KOMBAK

Marmara University, School of Medicine, Department of Pathology,

İSTANBUL, TURKEY

E-mail: erdemkombak@hotmail.com Phone: +90 5333793508 
chain reaction (RT-PCR) and Sanger sequencing are the gold standard techniques to detect BRAFV600E mutation, whereas immunohistochemistry (IHC) needs more scientific evidence of high specificity and sensitivity. Some morphological findings like multicentricity, lymph node metastasis, tumour extension beyond the thyroid parenchyma and Psammoma bodies (6) may also predict the BRAFV600E mutation.

CK19 is a low molecular weight cytokeratin found in simple and complex epithelia, as well as in some carcinomas. An increased intensity of CK19 immunostaining is used for the diagnosis of PTC. HBME-1 is a marker of the apical surface of the mesothelium. An apical membranous staining of HBME-1 is also seen in PTCs. Galectin-3 is a $\beta$-galactoside binding lectin in charge of cell adhesion. Nuclear and cytoplasmic immunostaining is seen in PTCs. Ki-67 detects the nuclei of cells in late G1, S, G2 and M phases. Proliferation index in PTCs is no more than $5 \%$ in general (7).

The aim of this study is to evaluate the immunoexpression of BRAFV600E, and its correlation with clinicopathologic parameters.

\section{MATERIALS and METHODS}

The study involved the use of formalin-fixed paraffinembedded tissue sections of histopathologically diagnosed cases of PTC $(n=107)$ and adenoma $(n=19)$ from the archives of the Department of Pathology. Of the PTC cases, 23 were microcarcinomas: 2 were follicular variants, 1 was oncocytic and 20 were classic. Twenty-three of the PTC group had metastatic lymph nodes available. These lymph nodes were assessed similarly. The slides that had been routinely stained with hematoxylin and eosin, CK19, HBME-1, Galectin-3 and Ki-67 were re-evaluated. The pT stage, necrosis, calcification, lymphatic invasion, vascular invasion, perineural invasion, tumour capsulation and capsule invasion, extrathyroidal extension, multicentricity, intrathyroidal spread and surgical margin status were assessed as prognostic parameters. To avoid controversy, certain criteria were used (8) as elaborated below.

Vascular invasion was defined as a direct tumour extension into the blood vessel lumen or a tumour aggregate within the vessel lumen. The criteria for vascular invasion are as follows:

- The affected vessel must be located within the capsule or immediately beyond the capsule but not within the tumour nodule itself.

- The vessel should have a clearly identifiable wall with endothelial lining.
- If a tumour extends directly into the vessel lumen, it should form a polypoid mass protruding into the lumen or exhibit thrombus formation in association with the tumour and not just bulging into the lumen.

- The cell aggregates within the lumen should be histologically identical to the tumour cells and be composed of epithelial cells and not of reactive endothelial cells.

- The intravascular tumour aggregate should be attached to the wall of the blood vessel and covered by a layer of endothelial cells.

Extrathyroidal extension was defined as tumour penetration through the thyroid pseudocapsule into the adjacent skeletal muscles or other organs.

Intrathyroidal spread was defined as an intraglandular dissemination of a tumour via lymphatic channels, and multiple small or larger satellite foci in the vicinity or remotely from the main tumour mass.

Information regarding the gender and age of the patients was obtained from the automation system of our hospital. Clinical follow-up was provided by the general surgery department. PTC cases were classified as either 'good prognosis (GP)' or 'poor prognosis (PP)' upon the clinical occurrence of lymph node metastasis, local recurrence and/ or distant metastasis. Clinicopathologic features are shown in Table I. Serial sections ( $4 \mu \mathrm{m}$ thick) were obtained from the paraffin-embedded blocks of the selected preparations and fixed on positively charged slides to perform IHC. BRAFV600E IHC was performed manually using the Novolink Polymer Detection System (Leica, Australia). Additional information on IHC is summarised in Table II. Positive and negative control slides were also stained. All 31 cases (14 primary tumours (PT), 13 metastatic lymph nodes and 4 adenomas) were selected randomly, and BRAF mutation analysis was performed on these cases using the Cobas 4800 RT-PCR System (Roche Diagnostics, USA).

\section{Assessment of Immunostaining}

The assessment of BRAFV600E IHC in PTCs, melanomas and colonic adenocarcinomas is still under debate (9). However, in our study, nuclear with or without cytoplasmic staining was considered positive as in most of the literature. A semiquantitative approach was used based on the staining intensity of positively stained cells: negative, $1+$ (weak staining), 2+ (moderate staining) and $3+$ (strong staining) of any proportion of tumour cells (Figures 1-4). Any proportion of tumour cells with membranous and cytoplasmic staining with CK19, apical 
membranous staining with HBME-1 and nuclear and/ or cytoplasmic staining with Galectin-3 was considered positive. The eyeballing technique was used for the Ki-67 labelling index, and 100 tumour cells were counted in hot spot areas. A proportion of nuclear-stained cells with Ki-67 was recorded and divided into groups with a threshold of $5 \%$.

\section{Statistical Analysis}

Statistical assessments were performed using the SPSS software (SPSS version 15, SPSS Inc., Chicago, IL, USA). Continuous variables were expressed as mean \pm standard deviation together with a range (minimum-maximum). The comparison of categorical variables was performed using the chi-square test. Fisher's exact test was used to compare BRAFV600E IHC and RT-PCR. A $P$-value of less than 0.05 was accepted as statistically significant.

Table I: Clinicopathologic features of the cases in the PTC group.

\begin{tabular}{|c|c|}
\hline Gender & \\
\hline Male & $22 \%(n=24)$ \\
\hline Female & $78 \%(n=83)$ \\
\hline Mean age & 44 years \\
\hline \multicolumn{2}{|l|}{ Type of resection } \\
\hline Total thyroidectomy & $44 \%(n=47)$ \\
\hline Lobectomy & $56 \%(n=60)$ \\
\hline \multicolumn{2}{|l|}{ Surgical margins } \\
\hline Positive & $23 \%(n=25)$ \\
\hline Negative & $77 \%(n=82)$ \\
\hline \multicolumn{2}{|l|}{ Clinical follow-up } \\
\hline Available & $91 \%(n=97)$ \\
\hline $\mathrm{N} / \mathrm{A}$ & $9 \%(n=10)$ \\
\hline Mean follow-up time & 25 months \\
\hline \multicolumn{2}{|l|}{ Survival } \\
\hline Alive & $99 \%(n=106)$ \\
\hline Dead & $1 \%(n=1)$ \\
\hline \multicolumn{2}{|l|}{ Lymph node metastasis } \\
\hline Synchronous & $24 \%(n=26)$ \\
\hline Later & $1 \%(n=1)$ \\
\hline Local recurrence & $5 \%(n=5)$ \\
\hline Distant metastasis & $2 \%(n=2)$ \\
\hline \multicolumn{2}{|l|}{ Prognostic group } \\
\hline Good prognosis & $68 \%(n=73)$ \\
\hline Poor prognosis & $32 \%(n=34)$ \\
\hline
\end{tabular}

PTC: Papillary carcinoma of the thyroid.

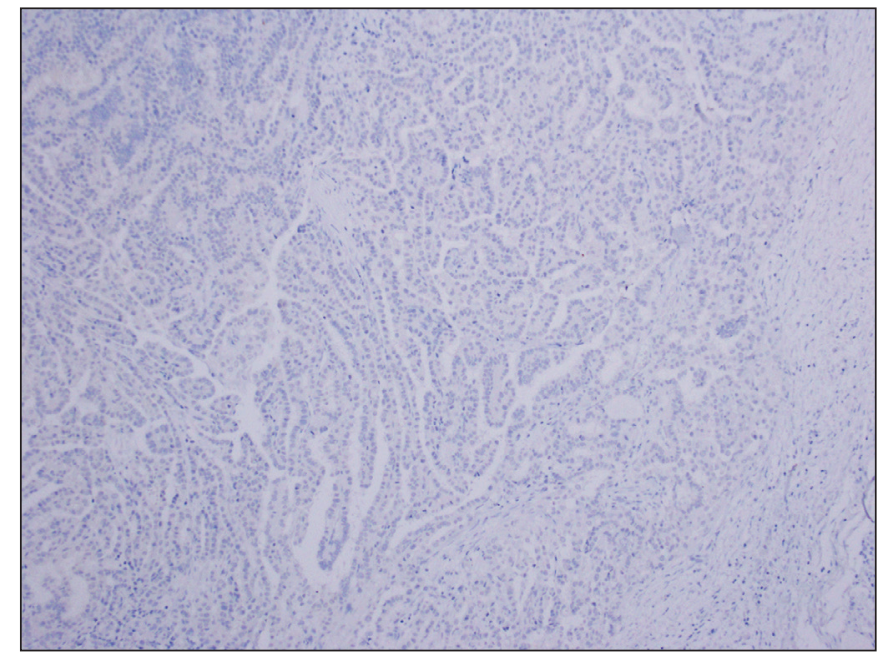

Figure 1: $B R A F V 600 \mathrm{E}$ IHC negative (x200).

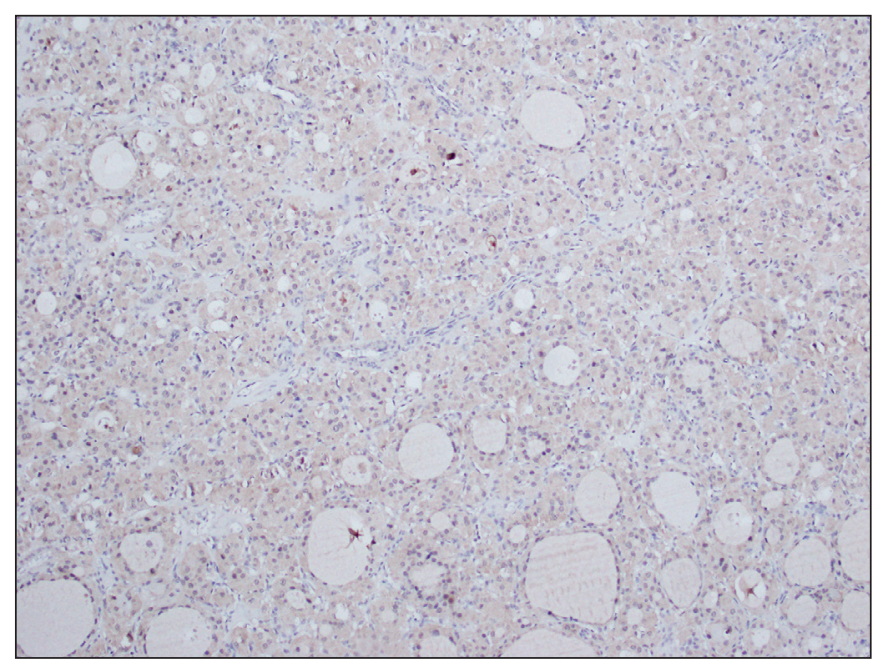

Figure 2: BRAFV600E IHC 1+ (x200).

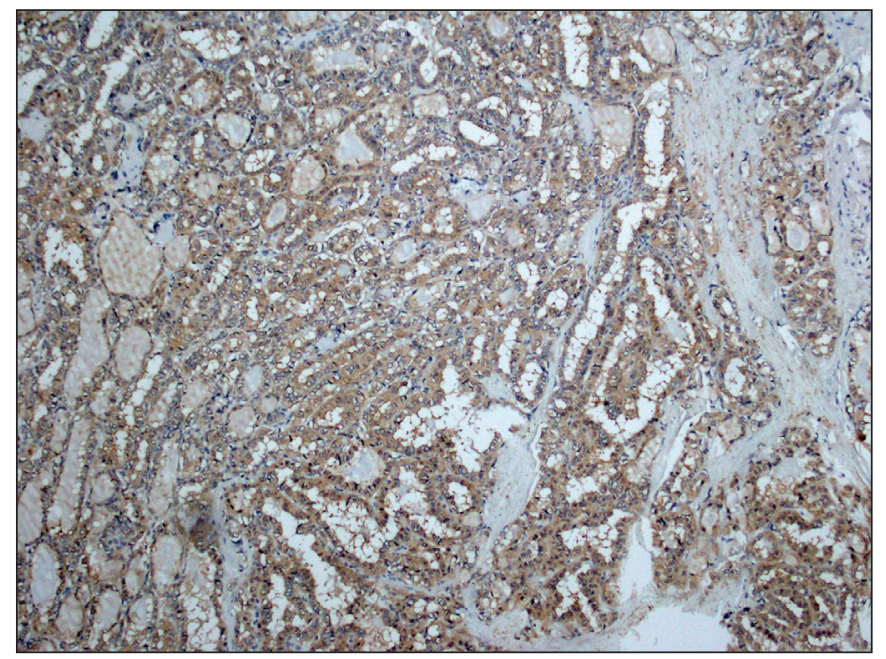

Figure 3: BRAFV600E IHC 2+ (x200). 
Table II: Details of immunohistochemical findings.

\begin{tabular}{llccc}
\hline Antibody & Clone & Dilution & Incubation time $(\mathbf{m i n})$ & Temperature $\left({ }^{\circ} \mathrm{C}\right)$ \\
\hline BRAFV600E & VE-1 $(\mathrm{mm}) ;$ Spring Bioscience & $1 / 100$ & 60 (manual) & 37 \\
\hline CK19 & AB53 (mm); Dako & $1 / 100$ & 30 (Bond-Max) & 56 \\
\hline HBME-1 & HBME-1 (mm); Dako & $1 / 50$ & 30 (Bond-Max) & 56 \\
\hline Galectin-3 & 9C4 $(\mathrm{mm}) ;$ Dako & $1 / 100$ & 30 (Bond-Max) & 56 \\
\hline Ki-67 & SP6 (rm); Abcam & $1 / 100$ & 30 (Bond-Max) & 56 \\
\hline
\end{tabular}

IHC: Immunohistochemistry, mm: Mouse monoclonal, rm: Rabbit monoclonal.

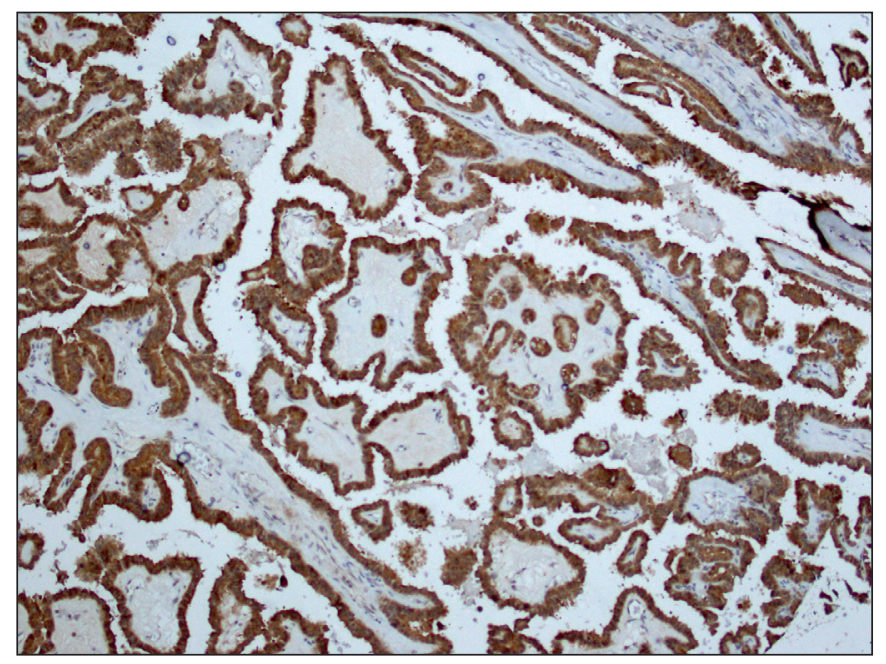

Figure 4: BRAFV600E IHC 3+ (x200).

\section{RESULTS}

Of the PTC cases, $43 \%(n=46)$ was pT1, 27\% $(n=29)$ pT2 and $30 \%(n=32) \mathrm{pT} 3$. The mean tumour size was $20.98 \mathrm{~mm}$ (min. $3 \mathrm{~mm}$ and max. $75 \mathrm{~mm}$ ) in PTCs and $25.79 \mathrm{~mm}$ (min. $9 \mathrm{~mm}$ and max. $67 \mathrm{~mm}$ ) in adenomas. Of the PTC cases, $45 \%$ $(n=48)$ was classic variant, $23 \%(n=25)$ follicular, $10 \%(n$ $=11)$ oncocytic and $22 \%(n=23)$ microcarcinomas (Figures 5-8). Of the microcarcinomas, $87 \%(n=20)$ was classic variant, $9 \%(n=2)$ follicular and $4 \%(n=1)$ oncocytic. Of the adenomas, $15.8 \%(n=3)$ was oncocytic.

Twelve of the PTC cases were negative for HBME-1, one was negative for CK19 and six were negative for Galectin-3. In BRAFV600E IHC, 31 of the PTC cases were negative. Twelve cases showed a $\mathrm{Ki}-67$ proliferation index higher than 5\%. For BRAFV600E IHC, positive cases exhibited varying percentages of staining as shown in Table III.

The results of BRAF RT-PCR of randomly selected cases are shown in Table IV. For comparison of IHC and RTPCR, the highest likelihood ratio (8.18) was obtained with the hypothesis 'Only $3+\mathrm{IHC}$ of $B R A F \mathrm{~V} 600 \mathrm{E}$ is truly positive'. The sensitivity of $B R A F \mathrm{~V} 600 \mathrm{E}$ IHC was calculated

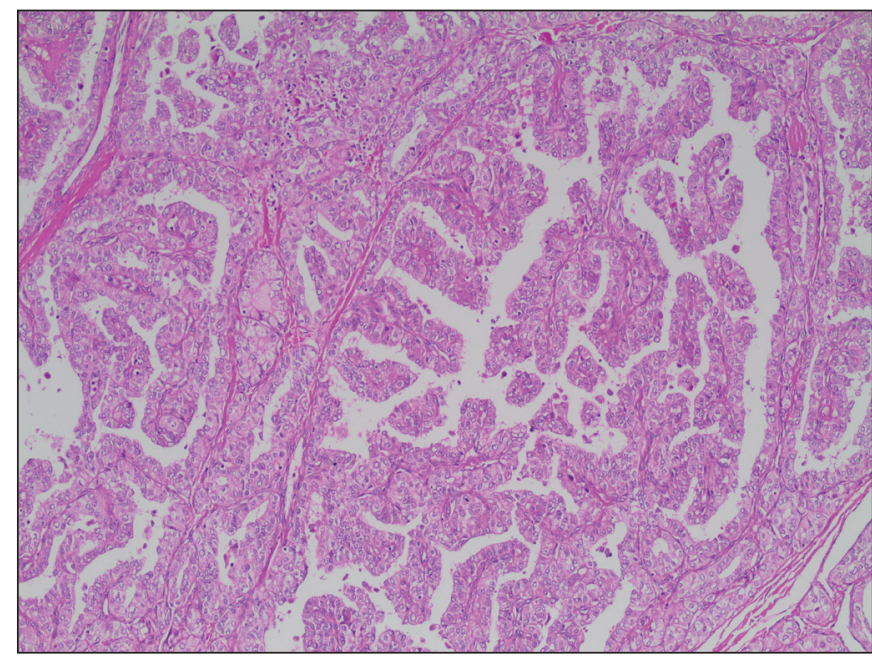

Figure 5: Classic variant papillary carcinoma (H\&E; x200).

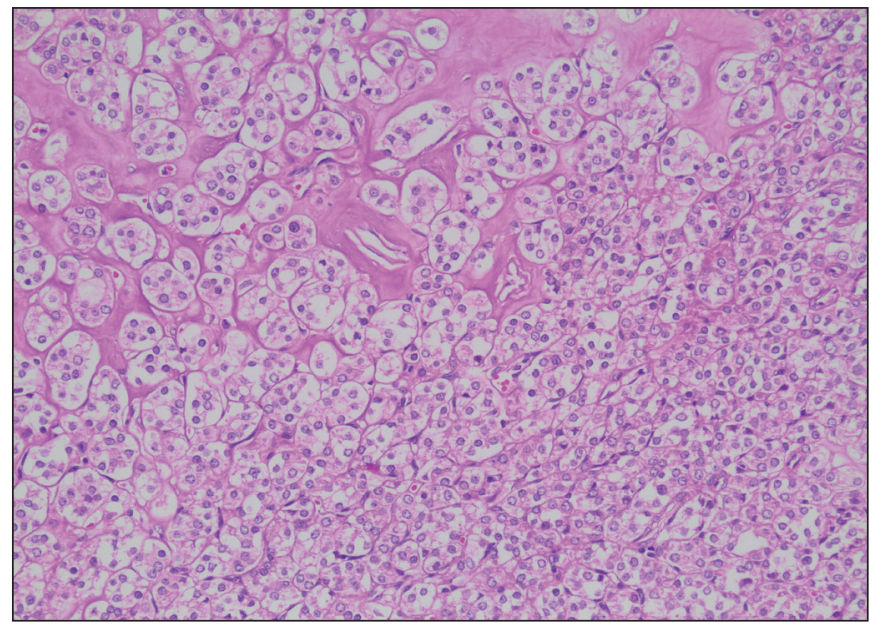

Figure 6: Follicular variant papillary carcinoma (H\&E; x200).

at $90.9 \%$, whereas the specificity was $88.8 \%$. In addition, the positive predictive value was $95.2 \%$, and the negative predictive value was $80 \%$. Given the likelihood ratio at $8.18,1$ of every 10 tests was meant to be wrong. In this respect, the BRAFV600E IHC findings are reconsidered and summarised in Table $\mathrm{V}$. 
Table III: BRAFV600E IHC.

\begin{tabular}{|c|c|c|c|c|c|}
\hline \multirow{2}{*}{ Group } & \multicolumn{5}{|c|}{$B R A F V 600 \mathrm{E}$ staining intensity $(n)$} \\
\hline & 0 & $1+$ & $2+$ & $3+$ & Total \\
\hline PTC-classic & 11 & 3 & 7 & 27 & 48 \\
\hline PTC-oncocytic & 2 & 5 & 3 & 1 & 11 \\
\hline Papillary microcarcinoma & 5 & 3 & 9 & 6 & 23 \\
\hline Normal thyroid tissue & 2 & 10 & 1 & 0 & 13 \\
\hline Total & 45 & 39 & 21 & 34 & 139 \\
\hline
\end{tabular}

IHC: Immunohistochemistry, PTC: Papillary carcinoma of the thyroid.

Table IV: BRAF RT-PCR results.

\begin{tabular}{lcccccc}
\hline & IHC $(-)$ & IHC $(\mathbf{1 + )}$ & IHC (2+) & IHC (3+) & Total & 1 \\
\hline RT-PCR $(-)$ & 10 & 5 & 5 & 1 & 8 & 10 \\
\hline RT-PCR $(+)$ & 1 & 0 & 5 & 6 & 9 & 31 \\
\hline Total & 11 & 5 & 1 & 9 \\
\hline
\end{tabular}

RT-PCR: Polymerase chain reaction, IHC: Immunohistochemistry.

Table V: BRAFV600E IHC upon RT-PCR results.

\begin{tabular}{lccc}
\hline \multirow{2}{*}{ Group } & \multicolumn{3}{c}{ BRAFV600E staining intensity $(\boldsymbol{n}(\mathbf{\%}))$} \\
\cline { 2 - 4 } & $\mathbf{0 / 1 + / 2 +}$ & $\mathbf{3 +}$ & Total \\
\hline PTC-classic & 21 & $27(59.5)$ & 48 \\
\hline PTC-follicular & 25 & $1(9)$ & 25 \\
\hline PTC-oncocytic & 10 & $6(26)$ & 11 \\
\hline PMC & 17 & $34(31.8)$ & 23 \\
\hline Total & 73 & 107 \\
\hline
\end{tabular}

IHC: Immunohistochemistry, RT-PCR: Polymerase chain reaction, PTC: Papillary carcinoma of the thyroid, PMC: Papillary microcarcinoma.

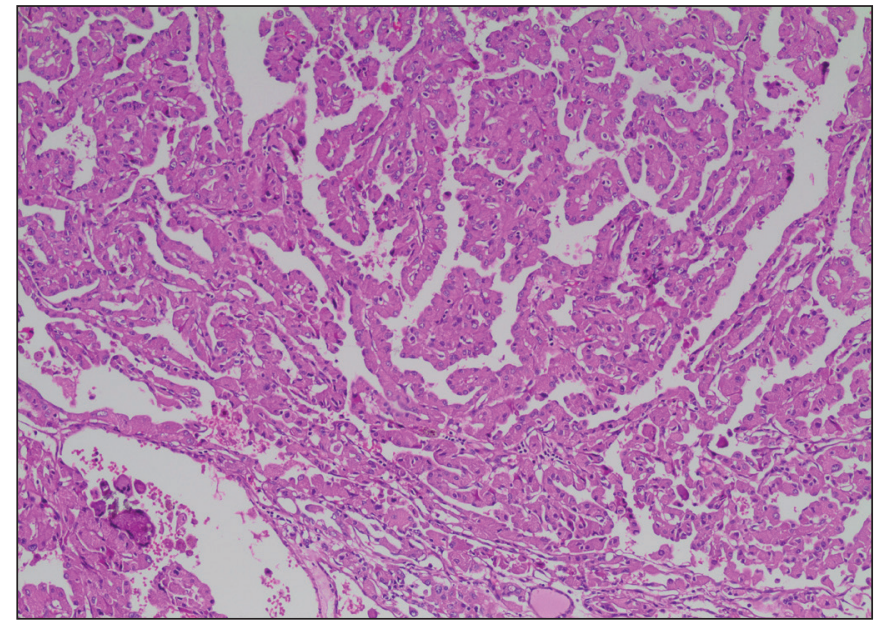

Figure 7: Oncocytic variant papillary carcinoma (H\&E; x200).

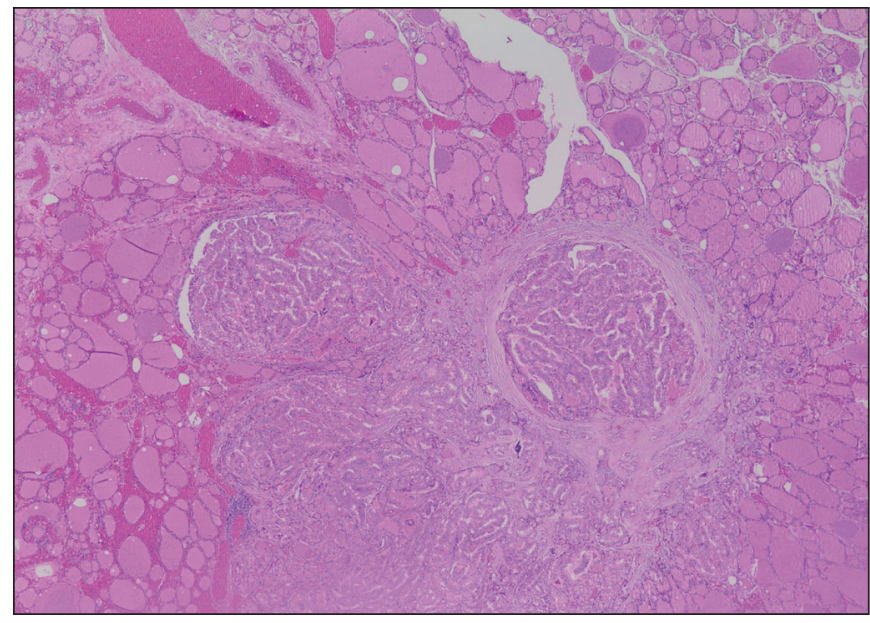

Figure 8: Papillary microcarcinoma (H\&E; x40). 
In cases where a metastatic lymph node (MLN) is present, the BRAFV600E positivity was $11 \%$ in PT. Two of those showed no staining in MLN, whereas PT was positive. Another two cases showed a lower percentage of positive tumour cells in MLN (Table VI). However, PTs and conjugate MLNs were statistically correlated upon the $B R A F V 600 \mathrm{E}$ mutation $(\mathrm{Phi}=83.7 \%, p=0.0001)$.

The BRAFV600E mutation was found to be statistically correlated with a higher $\mathrm{pT}$ stage $(p=0.003)$, classic morphology $(p=0.003)$, lower mitotic index $(p=0.020)$, lymphatic invasion $(p=0.013)$, perineural invasion $(p=$ $0.006)$, a lack of tumour capsule $(p=0.016)$, extrathyroidal extension $(p=0.0001)$ and intrathyroidal spread $(p=$ 0.0001 ). Noassociation was found between the BRAFV600E mutation and the patient's age, sex, synchronous lymph node metastasis, necrosis, calcification, vascular invasion, tumour capsule invasion, multicentricity, expressions of CK19, HBME-1, and Galectin-3 and the Ki-67 proliferation index. Of the BRAFV600E-mutated cases, $52.9 \%$ exhibited nodular hyperplasia in the non-tumoural parenchyma, whereas $23.5 \%$ showed lymphocytic thyroiditis. Neither had a significant association.

The clinical prognosis was assessed in two separate groups as described earlier. The poor prognostic group was found to be statistically correlated with a higher pT stage $(p=$ $0.005)$, classic morphology $(p=0.011)$, calcification $(p=$ $0.017)$, lymphatic invasion ( $p=0.008)$, vascular invasion $(p$ $=0.0001)$, lack of tumour capsule $(p=0.004)$, extrathyroidal extension $(p=0.0001)$, intrathyroidal spread $(p=0.001)$ and positive surgical margin $(p=0.002)$. No association was found with the patient's age, sex, necrosis, mitotic index, perineural invasion, tumour capsule invasion and multicentricity. HBME-1 positive cases were found to be correlated with PP $(p=0.049)$, whereas CK19 expression, Galectin-3 expression and Ki-67 proliferation index were irrelevant.

Table VI: BRAFV600E positive cells (3+ intensity) in PTs and conjugate MLNs.

\begin{tabular}{|c|c|c|c|c|}
\hline Case & PT-BRAF & PT-BRAF-\% & MLN-BRAF & MLN-BRAF-\% \\
\hline 1 & Negative & & Negative & \\
\hline 2 & Positive & 90 & Positive & 90 \\
\hline 3 & Positive & 80 & Positive & 70 \\
\hline 4 & Negative & & Negative & \\
\hline 5 & Positive & 100 & Negative & \\
\hline 6 & Negative & & Negative & \\
\hline 7 & Negative & & Negative & \\
\hline 8 & Positive & 100 & Positive & 100 \\
\hline 9 & Negative & & Negative & \\
\hline 10 & Positive & 90 & Positive & 40 \\
\hline 11 & Negative & & Negative & \\
\hline 12 & Negative & & Negative & \\
\hline 13 & Negative & & Negative & \\
\hline 14 & Negative & & Negative & \\
\hline 15 & Positive & 100 & Positive & 100 \\
\hline 16 & Negative & & Negative & \\
\hline 17 & Positive & 90 & Positive & 90 \\
\hline 18 & Positive & 100 & Positive & 100 \\
\hline 19 & Negative & & Negative & \\
\hline 20 & Positive & 100 & Positive & 100 \\
\hline 21 & Positive & 100 & Positive & 100 \\
\hline 22 & Positive & 100 & Negative & \\
\hline 23 & Negative & & Negative & \\
\hline
\end{tabular}

PT-BRAF: BRAFV600E mutation in primary tumour, PT-BRAF-\%: Percentage of BRAFV600E positive cells in primary tumour, MLN-BRAF: BRAFV600E mutation in conjugate metastatic lymph node, MLN-BRAF-\%: Percentage of BRAFV600E positive cells in conjugate metastatic lymph node, PT: Primary tumour, MLN: Metastatic lymph node. 
In cases where the $B R A F \mathrm{~V} 600 \mathrm{E}$ mutation was present, poor prognostic incidents such as lymph node metastasis, local recurrence and distant metastasis were more frequent. However, we could not reveal any statistical significance $(p=0.255)$. On the other hand, BRAFV600E positive cases constituted a minority of $40 \%$ in the poor prognostic group.

\section{DISCUSSION}

PTCs are malignant tumours with an increasing incidence rate (1). The widespread use of radiological techniques and fine-needle aspiration biopsy has been partly responsible for this increasing incidence, although the vast majority of new cases are microcarcinomas (10). Lobectomy or total thyroidectomy with subsequent radioactive iodine ablation in selected patients is usually more effective; however, approximately $10 \%$ of PTC patients suffers from recurrence, lymph node or distant metastasis and hence require further intervention. BRAF mutations are the major promising development for PTC recently, providing a highly efficient anti-tyrosine kinase therapy.

Several studies have compared BRAFV600E IHC with molecular techniques. For instance, Qiu et al. (11) assessed BRAFV600E IHC by distinguishing samples as positive and negative without considering staining intensity and percentage of stained tumour cells. The study also compared IHC with RT-PCR and Sanger sequencing. Jung et al. (12) also compared IHC with RT-PCR and BRAF RNA in situ hybridisation. Zagzag et al. (13) accepted $3+$ BRAFV600E staining as positive and compared IHC with direct sequencing. Ilie et al. (14) accepted the results as positive if $100 \%$ of the tumour cells stained $3+$ and compared IHC with direct sequencing. To sum up, the sensitivity and specificity of BRAFV600E IHC ranges from $89 \%$ to $100 \%$ and from $61 \%$ to $100 \%$, respectively. In our study, we accepted 3+ BRAFV600E staining as positive, disregarding the percentage of tumour cells, and compared IHC with RT-PCR. The sensitivity of BRAFV600E IHC was $90.9 \%$, whereas the specificity was $88.8 \%$. It is important to note that the RT-PCR system we used detects V600D and V600K mutations along with V600E, thus giving a nondiscriminatory result.

Using the criteria $3+$ nuclear and cytoplasmic staining in the PTC group, the BRAFV600E mutation rate was $31.8 \%$. This rate increased up to $59.5 \%$ in classic variant cases, but it decreased to $26 \%$ in papillary microcarcinomas (PMCs) and $9 \%$ in oncocytic variant cases. In follicular variant and adenoma cases, no mutation was detected. In the recent literature, the BRAFV600E mutation rate has been reported between $35 \%$ and $70 \%$, and the mutations were more often associated with a classic variant, tall cell variant and poorly differentiated/anaplastic carcinomas that arise from welldifferentiated PTCs (15). The mutation rate is much lower in follicular carcinomas (16), which is similar to our results.

Intratumoural heterogeneity is a substantial phenomenon for understanding pathogenesis and its clinicopathologic role. As in other BRAF-harbouring tumours such as malignant melanomas and colorectal and pulmonary adenocarcinomas, PTCs have been shown to exhibit heterogeneously mutated tumour cells. Guerra et al. (17) showed BRAF-mutated tumour cells in MLNs of cases with $B R A F$ negative primary, prompting that $B R A F$ mutations constitute a subclonal alteration and may arise de novo in $B R A F$ negative tumours later on. On the other hand, de Biase et al. (16) revealed a direct proportion between tumour size and percentage of $B R A F$-mutated tumour cells, suggesting that $B R A F$ mutation is an early period alteration. Walts et al. (18) stated $100 \%$ concordance of BRAF mutation between PT and MLN and 92.3\% concordance between different areas of PTs. In their experience, two $B R A F$-mutated PT cases exhibited BRAF-negative MLNs and recurrent tumours afterward. We observed a range of $80-90 \%$ BRAF-mutated tumour cells in PTs, two of which exhibited $B R A F$-negative MLNs and the other two showed a lower percentage of $B R A F$-mutated tumour cells in conjugated MLNs. The existence of such subclones disturbs the efficacy of targeted therapies. In this regard, quantitative $B R A F$ mutation analysis may be suggested in PT, MLN, distant metastasis or recurrent tumour samples.

To start with associations between the BRAFV600E mutation and clinicopathologic parameters, we found noassociation with the patient's age and sex, as in the largescale meta-analysis of Wang et al. (19) and series of Shin et al. (20). In our experience, BRAFV600 mutation was found to be correlated with a higher pT stage, lymphatic invasion, perineural invasion, lack of tumour capsule, extrathyroidal extension and intrathyroidal spread. Several studies have stated various morphological findings, and their combinations are correlated with the BRAFV600E mutation, interestingly having extrathyroidal extension in common $(6,21,22)$.

Surprisingly, the BRAFV600E mutation rate was higher in tumours with a lower mitotic index, as in tumours with a lower Ki-67 proliferation index, despite its incoherency. No effort has been found in the English literature that addresses theassociation between BRAF mutations and mitotic index or the Ki-67 proliferation index. Nevertheless, welldifferentiated PTCs are known to have a lower proliferation index than other malignancies. We observed that the Ki-67 
proliferation index is higher than $5 \%$ in $19.6 \%$ of the PTC cases, reaching up to $15 \%$. In addition, we did not find any significant association between mitotic/Ki-67 index and worse clinical and/or pathologic prognostic parameters. Guerra et al. (23) showed a higher rate of CK19 expression in BRAF-mutated tumours, whereas Galectin-3 was not associated with $B R A F$. In terms of HBME- 1 expression and $B R A F$, our effort needs to be published first. However, in our series, no significantassociation was found between the BRAFV600E mutation and expression of CK19, Galectin-3 and HBME-1.

In cases where follow-up data are available, a survival analysis could not be made because there was no death by disease. The cases were assessed in two separate groups: GP and PP, as described earlier. The patient's age and sex were not found to be correlated with the prognosis. This is despite the fact that Howell et al. (24) stated that the BRAFV600E mutation and older age ( $\geq 65$ years) predict recurrence and Suman et al. (25) associated younger age ( $\leq$ 45 years) with central lymph node metastasis.

In our series, $\mathrm{PP}$ was found to be associated with a higher $\mathrm{pT}$ stage, classic morphology, calcification, lymphatic invasion, vascular invasion, lack of tumour capsule, intrathyroidal spread, extrathyroidal extension, positive surgical margin and loss of HMBE-1 expression. Likewise, Rossi et al. (26) have associated PP in poorly differentiated and anaplastic thyroid carcinomas with loss of HBME-1 expression.

The association between the BRAFV600E mutation and PP can be properly summarised by the meta-analysis of Wang et al. (19). In contrast to what has been reported recently. Pelttari et al. (27), with their lengthy follow-up duration, have shown that the BRAFV600E mutation is not correlated with lymph node metastasis and/or recurrence. Zheng et al. (28) have revealed that the BRAFV600E mutation and the recurrence within PMCs are not related. Nam et al. (21) have also shown that the BRAFV600E mutation is not significantly associated with lymph node metastasis. In these series, despite the overall concern, some morphological findings such as extrathyroidal extension are interestingly correlated with the BRAFV600E mutation. For instance, Shin et al. (20) revealed that the BRAFV600E mutation does not seem to be associated with the overall prognosis but morphological parameters are associated solely and together with aggressive behaviour. We also did not find any association between the BRAFV600E mutation and the overall prognosis but with such morphologic parameters.

In conclusion, BRAFV600E IHC with VE1 clone can be accepted as a reliable technique for detecting the
BRAFV600E mutation. Our series of well-differentiated PTCs has exhibited a rate of BRAFV600E mutation similar to recent literature. With our effort, morphological findings may be considered keener than the BRAFV600E mutation in predicting aggressive behaviour. However, demographic, clinical and morphological findings and genetic alterations should be assessed together to estimate a more precise prognosis. Although further therapeutic interventions are needed, it is better to look for the BRAFV600E mutation in PT, lymph node metastasis, recurrent tumour and distant metastasis, if available.

\section{ACKNOWLEDGEMENT}

We would like to express our sincere gratitude to Prof. Nural Bekiroğlu (Marmara University, School of Medicine, Department of Biostatistics) for her supervision in our statistical assessments.

\section{CONFLICT of INTEREST}

All authors declare that there is no conflict of interest that could be perceived as prejudicing the impartiality of the research reported.

\section{FUNDING}

This work was supported by the BAP (Scientific Research Projects Unit of Marmara University, grant number SAGC-TUP-121114-0357).

\section{REFERENCES}

1. Lim H, Devesa SS, Sosa JA, Check D, Kitahara CM. Trends in Thyroid Cancer Incidence and Mortality in the United States, 1974-2013. JAMA. 2017;317:1338-48.

2. Lloyd RV, Osamura RY, Klöppel G, Rosai J, Bosman FT, Jaffe ES, Lakhani SR, Ohgaki H. WHO Classification of Tumours of Endocrine Organs. 4th ed. Lloyd RV, Osamura RY, Klöppel G, Rosai J, editors. Lyon: International Agency for Research on Cancer; 2017.

3. Xing M, Alzahrani AS, Carson KA, Shong YK, Kim TY, Viola D, Elisei R, Bendlová B, Yip L, Mian C, Vianello F, Tuttle RM, Robenshtok E, Fagin JA, Puxeddu E, Fugazzola L, Czarniecka A, Jarzab B, O’Neill CJ, Sywak MS, Lam AK, Riesco-Eizaguirre G, Santisteban P, Nakayama H, Clifton-Bligh R, Tallini G, Holt EH, Sýkorová V. Association between BRAF V600E mutation and recurrence of papillary thyroid cancer. J Clin Oncol. 2015;33:4250.

4. Niederer-Wust SM, Jochum W, Forbs D, Brandle M, Bilz S, Clerici T, Oettli R, Müller J, Haile SR, Ess S, Stoeckli SJ, Broglie MA. Impact of clinical risk scores and BRAF V600E mutation status on outcome in papillary thyroid cancer. Surgery. 2015;157:11925.

5. Li F, Chen G, Sheng C, Gusdon AM, Huang Y, Lv Z, Xu H, Xing $\mathrm{M}, \mathrm{Qu}$ S. BRAFV600E mutation in papillary thyroid microcarcinoma: A meta-analysis. Endocr Relat Cancer. 2015;22:159-68. 
6. Virk RK, Theoharis CG, Prasad A, Chhieng D, Prasad ML. Morphology predicts BRAFV600E mutation in papillary thyroid carcinoma: An interobserver reproducibility study. Virchows Arch. 2014;464:435-42.

7. Kjellman P, Wallin G, Hoog A, Auer G, Larsson C, Zedenius J. MIB-1 index in thyroid tumors: A predictor of the clinical course in papillary thyroid carcinoma. Thyroid. 2003;13:371-80.

8. Nikiforov YE, Biddinger PW, Thompson LDR. Diagnostic Pathology and Molecular Genetics of the Thyroid Philadelphia: Wolters Kluwer; 2015. Available from: http:/qut.eblib.com.au/ patron/FullRecord.aspx?p=3417856.

9. Pyo JS, Sohn JH, Kang G. BRAF Immunohistochemistry Using Clone VE1 is Strongly Concordant with BRAF(V600E) Mutation Test in Papillary Thyroid Carcinoma. Endocrine pathology. 2015;26:211-7.

10. Burgess JR, Tucker P. Incidence trends for papillary thyroid carcinoma and their correlation with thyroid surgery and thyroid fine-needle aspirate cytology. Thyroid. 2006;16:47-53.

11. Qiu T, Lu H, Guo L, Huang W, Ling Y, Shan L, Li W, Ying J, Lv N. Detection of BRAF mutation in Chinese tumor patients using a highly sensitive antibody immunohistochemistry assay. Scientific reports. 2015;5:9211.

12. Jung YY, Yoo JH, Park ES, Kim MK, Lee TJ, Cho BY, Chung YJ, Kang KH, Ahn HY, Kim HS. Clinicopathologic correlations of the BRAFV600E mutation, BRAF V600E immunohistochemistry, and BRAF RNA in situ hybridization in papillary thyroid carcinoma. Pathology, research and practice. 2015;211:162-70.

13. Zagzag J, Pollack A, Dultz L, Dhar S, Ogilvie JB, Heller KS, Deng FM, Patel KN. Clinical utility of immunohistochemistry for the detection of the BRAF v600e mutation in papillary thyroid carcinoma. Surgery. 2013;154:199-204; discussion 1204-5.

14. Ilie MI, Lassalle S, Long-Mira E, Bonnetaud C, Bordone O, Lespinet V, Lamy A, Sabourin JC, Haudebourg J, Butori C, Guevara N, Peyrottes I, Sadoul JL, Bozec A, Santini J, Capper D, von Deimling A, Emile JF, Hofman V, Hofman P. Diagnostic value of immunohistochemistry for the detection of the BRAF(V600E) mutation in papillary thyroid carcinoma: Comparative analysis with three DNA-based assays. Thyroid. 2014;24:858-66.

15. Nikiforova MN, Kimura ET, Gandhi M, Biddinger PW, Knauf JA, Basolo F, Zhu Z, Giannini R, Salvatore G, Fusco A, Santoro M, Fagin JA, Nikiforov YE. BRAF mutations in thyroid tumors are restricted to papillary carcinomas and anaplastic or poorly differentiated carcinomas arising from papillary carcinomas. J Clin Endocrinol Metab. 2003;88:5399-404.

16. de Biase D, Cesari V, Visani M, Casadei GP, Cremonini N, Gandolfi G, Sancisi V, Ragazzi M, Pession A, Ciarrocchi A, Tallini G. High-sensitivity BRAF mutation analysis: BRAF V600E is acquired early during tumor development but is heterogeneously distributed in a subset of papillary thyroid carcinomas. J Clin Endocrinol Metab. 2014;99:E1530-8.
17. Guerra A, Sapio MR, Marotta V, Campanile E, Rossi S, Forno I, Fugazzola L, Budillon A, Moccia T, Fenzi G, Vitale M. The primary occurrence of $\mathrm{BRAF}(\mathrm{V} 600 \mathrm{E})$ is a rare clonal event in papillary thyroid carcinoma. J Clin Endocrinol Metab. 2012;97:517-24.

18. Walts AE, Pao A, Sacks W, Bose S. BRAF genetic heterogeneity in papillary thyroid carcinoma and its metastasis. Hum Pathol. 2014;45:935-41.

19. Wang Z, Chen JQ, Liu JL, Qin XG. Clinical impact of BRAF mutation in the diagnosis and prognosis of papillary thyroid carcinoma: A systematic review and meta-analysis. Eur J Clin Invest. 2016;46:146-57.

20. Shin MK, Kim JW, Min SK, Lee DJ, Kim JH, Lee SC, Chung BW, Ju YS. Associations of the BRAF (V600E) mutation and p53 protein expression with clinicopathological features of papillary thyroid carcinomas patients. Oncol Lett. 2015;10:1882-8.

21. Nam JK, Jung CK, Song BJ, Lim DJ, Chae BJ, Lee NS, Park WC, Kim JS, Jung SS, Bae JS. Is the BRAF(V600E) mutation useful as a predictor of preoperative risk in papillary thyroid cancer? Am J Surg. 2012;203:436-41.

22. Howell GM, Nikiforova MN, Carty SE, Armstrong MJ, Hodak SP, Stang MT, McCoy KL, Nikiforov YE, Yip L. BRAF V600E mutation independently predicts central compartment lymph node metastasis in patients with papillary thyroid cancer. Ann Surg Oncol. 2013;20:47-52.

23. Guerra A, Marotta V, Deandrea M, Motta M, Limone PP, Caleo A, Zeppa P, Esposito S, Fulciniti F, Vitale M. BRAF (V600E) associates with cytoplasmatic localization of p27kip1 and higher cytokeratin 19 expression in papillary thyroid carcinoma. Endocrine. 2013;44:165-71.

24. Howell GM, Carty SE, Armstrong MJ, Lebeau SO, Hodak SP, Coyne C, Stang MT, McCoy KL, Nikiforova MN, Nikiforov YE, Yip L. Both BRAF V600E mutation and older age $(>/=65$ years) are associated with recurrent papillary thyroid cancer. Ann Surg Oncol. 2011;18:3566-71.

25. Suman P, Wang CH, Abadin SS, Moo-Young TA, Prinz RA, Winchester DJ. Risk factors for central lymph node metastasis in papillary thyroid carcinoma: A National Cancer Data Base (NCDB) study. Surgery. 2016;159:31-40.

26. Rossi ED, Straccia P, Palumbo M, Stigliano E, Revelli L, Lombardi CP, Santeusanio G, Pontecorvi A, Fadda G. Diagnostic and prognostic role of HBME-1, galectin-3, and beta-catenin in poorly differentiated and anaplastic thyroid carcinomas. Appl Immunohistochem Mol Morphol. 2013;21:237-41.

27. Pelttari H, Schalin-Jantti C, Arola J, Loyttyniemi E, Knuutila S, Valimaki MJ. BRAF V600E mutation does not predict recurrence after long-term follow-up in TNM stage I or II papillary thyroid carcinoma patients. APMIS. 2012;120:380-6.

28. Zheng X, Wei S, Han Y, Li Y, Yu Y, Yun X, Ren X, Gao M. Papillary microcarcinoma of the thyroid: Clinical characteristics and BRAF(V600E) mutational status of 977 cases. Ann Surg Oncol. 2013;20:2266-73. 